\title{
Using Total Probability in Image Template Matching
}

\author{
Haval Sadeq \\ College of Engineering, Salahaddin University-Erbil, Erbil-Iraq
}

\begin{abstract}
Image template matching is a main task in photogrammetry and computer vision. The matching can be used to automatically determine the $3 D$ coordinates of a point. A firstborn image matching method in fields of photogrammetry and computer vision is area-based matching, which is based on correlation measuring that uses normalised cross-correlation. However, this method fails at a discontinuous edge and at the area of low illumination or at geometric distortion because of changes in imaging location. Thus, these points are considered outliers. The proposed method measures correlations, which is based on normalised cross-correlation, at each point by using various sizes of window and then considering the probability of correlations for each window. Thereafter, the determined probability values are integrated. On the basis of a specific threshold value, the point of maximum total probability correlation is recognised as a corresponding point. The algorithm is applied to aerial images for Digital Surface Model (DSM) generation. Results show that the corresponding points are identified successfully at different locations, especially at a discontinuous point, and that a Digital Surface Model of high resolution is generated.
\end{abstract}

Keywords: Digital surface model, template image matching, normalised cross-correlation, probability.

Received February 11, 2018; accepted June 11, 2019

https://doi.org/10.34028/iajit/17/3/8

\section{Introduction}

An important tool that affects the result of the products of digital photogrammetry, image analysis and computer vision is image matching. In photogrammetry, the result of image matching, which is also known as stereo matching, is used to find the parallax that can be used to find the 3D coordinates of a point by using collinearity equations. Although different techniques have been developed for image matching since its introduction in the 1950s [13], the field is still considered an active research area because no robust technique or algorithm can be used to find conjugate points robustly. The failure of image matching is due to various reasons, such as lack of sufficient texture, breaklines or gaps in objects, nonplanarity in local objects, confusion because of repetitive patterns, occlusions, shifted objects, shadows, multi-layered objects and reflections and artefacts because of radiometric effect [24, 47]. Image matching has various applications. For example, it is used for tie point determination in relative orientation and Digital Surface Model (DSM) generation [9, 30, 47]. It can also be used in image registration [11, 38, 42, 49].

Image matching principles are classified into three main categories, namely, intensity- or area-based matching, feature-based matching [16] and a combination of the two methods or hybrid or relational matching [13, 24, 25, 39]. Intensity-based matching is used to search for areas of similar radiometric grey values in two or more images. It is classified into two methods depending on the implemented technique: one is cross-correlation optimisation and least square matching; the other is Semi-Global Matching (SGM), which focuses on using mutual informational hierarchically to find the corresponding points [17].

At the same time, feature-based matching is based on finding the corresponding points by using features. Although different algorithms are available for feature-based matching, the popular methods are least square matching of features, clustering, dynamic programming and Scale-Invariant Feature Transform (SIFT) matching [24].

\section{Related Works}

Numerous studies have focused on image matching using cross-correlation algorithm, which is implemented in producing DSMs $[4,27,33,44,46$, 47]. The algorithm was used by Xie [40] for 3D object production from satellite images for web-based visualisation. Normalised Cross-Correlation (NCC) is used to aid candidate matching for image matching [29]. Dall'Asta and Roncella [6] compared NCC with other image matching algorithms such as sum of absolute/squared differences, census transform method for surface construction and SGM. Debella and Kaab [7] used NCC by locally adopting an optimisation of template size for mass shift by determining its horizontal surface movement. It is also used in image registration [2]. It is also applied to detect the shift of two datasets (e.g., the ASTER GDEM2 relative to the DEM of Geographical Survey Institute of Japan) [35]. NCC has been used by to measure the similarity of the 
features that extracted from the watermarked image and the original cover image [32].

Zhang et al. [45] used NCC by integrating with edge matching for accurate DSM generation. NCC is used by Höhle and Potuckova [18] to find the corresponding points up to pixel accuracy, which was later refined to sub-pixel accuracy by using least square matching. Hong and Zhang [19] implemented cross-correlation matching with relaxation-based matching and waveletbased feature extraction technique for image registration.

Different algorithms have been used to overcome the problems of cross-correlation. Gruen [12] implemented adaptive least squares by using basic model estimation to perform matching operation. An et al. [1] implemented normalised mutual information to be used in image matching. It is also used to find matches between two points on uncalibrated images and those that are specified to be invariant to the scale and rotation [48]. Chen et al. [5] modified NCC to find the corresponding terrestrial image accurately. Joglekar and Gedam [21] used zero mean NCC to generate a dense disparity map. Yang et al. [41] showed that variable NCC presents better results for image registration in quality and speed than does normalised crosscorrelation, normalised mutual information and correlation ration. The computation time of NCC has been reduced by determining the mean of the reference and the template in advance [26].

In the current research, the proposed approach is based on finding conjugate points by using various sizes of window. At each size of window, the values of NCC are converted into probability values. Then, the total probability value is obtained by using the product of the obtained probability value. Thus, the corresponding point is represented by the point of the maximum probability value. Aerial and satellite images are specified to be limited to one stereo pair; this value is rarely high. Therefore, this algorithm is used to find the corresponding points that are necessary to generate a DSM.

The rest of the paper is organised as follows. Firstly, image quality and how Signal-To-Noise Ratio (SNR) is used to measure it are discussed. Then, the NCC algorithm is illustrated, along with its use in comparing patches in stereo images. Thereafter, the proposed algorithm is introduced, followed by the test and assessment of the algorithm at different test areas. Lastly, the results are elaborated and presented.

\section{Image Quality}

In digital images, in addition to geometric rays that are emitted by the objects, radiometric information also contributes to the image formation [23]. The radiation that is received by the electro-optical detectors is exposed to noise during the conversion of lights into signals through the sensor $[34,37]$. The noise in an image affects its quality. Specifically, high noise means low quality. Each image pixel value $g_{i, j}$ is contaminated by the noise at locations $i$ and $j$. The true grey pixel value in the image at the same location is represented by $\breve{g}_{i, j}$, as shown in Equation (1).

$$
g_{i, j}=\bar{g}_{i, j}+N_{i, j}
$$

The noise in the image can be generally classified into three categories based on the source that embraces it [22]. Photon noise is generated because of the effect of randomly distributed photons when falling on the detector. During image acquisition, the number of photons that are recorded on the image is absolutely associated with the noise. In the feature that is specified to be bright, the noise is low because a large number of photons are emitted. By contrast, when few photons are emitted, the noise will be high. The second type is temperature noise or dark current noise. This type is produced because of collected photons at the detector and arising thermal factors, thereby leading to noise that is added to the original signal. The third type is known as detector (circuit) noise. This type is mainly produced because of the uncertainty in recovering the zero value when amplifying the pixel charge to a suitable level. The discussion above indicates that the total noise mainly depends on the type of the sensor and working environment. However, in most sensors, the total noise in the image can be summed up as shown in Equation (2).

$$
N=N_{\text {shot }}+N_{\text {dark-current }}+N_{\text {circuit }}
$$

The noise degrades image quality, thereby significantly affecting the efficiency of image matching. Different indices are used to assess the quality of image, namely, mean absolute error, mean square error, root mean square error and SNR [3]. A popular index for measuring the quality of image is SNR, which is discussed in detail in section 4 [34].

\section{Signal-to-Noise Ratio (SNR)}

Image quality significantly affects the result of image matching using area-based techniques. As mentioned in section 3, each pixel in the image is contaminated with noise (Equation (1)). Debella and Kaab [7] showed that SNR is represented by the ratio of the electric signal variance $(S)$ to the noise variance $(N)$ Equation (3).

$$
S N R=\frac{\sigma^{2}(S)}{\sigma^{2}(N)}
$$

The value of SNR can be used as radiometric sensitivity across the spectrum. When the value of electronic signal equals the value of noise, the value of SNR is unity. The value of SNR can reach 1000 or higher [23]. High value of SNR indicates that the electronic signals dominate over the noise; thus, the 
geometric object feature can be clearly identified. The feature is considered important in extracting geometric objects from images. In the case of shadows, noise is predominated, thereby leading to low value of SNR; thus, the basic geometric feature is not visible, and either the feature cannot be extracted or the objects are faintly extracted; moreover, some of the extracted edges are broken [20].

For stereo image matching, as suggested by Zhang [43], to increase the size of window in the locations that is specified with low value of SNR (e.g., in shadowed and sloped areas) to include a large number of features and thus increase the variance. However, this idea is not applicable in uniform textures over large areas. The size of window is important in locations wherein the windows cover the edge of the building. Increasing the size of window exerts an important effect on the variance by including an edge in the window, thereby leading to an increase in the variance of the signal and consequently increasing the SNR because of highintensity variation. SNR is increased because of contrast in texture, thereby leading to diverse pixel values. This process leads to increased variance, but this is not always the case. In homogeneous surfaces or shadowed areas, even increasing the size of window does not affect the value of SNR because including a large number of pixels in the window will lead to including the same amount of noise. Occasionally, small size of window functions extremely effectively fails at low value of SNR. The mismatch problem can often occur at locations of low texture or shadow. Similarity in pixels or repetitive structures can lead to failure in image matching. Consequently, this process results in a challenge even when using large sizes of window

\section{Normalised Cross Correlation (NCC)}

The aim of applying automatic image matching is to find the corresponding or conjugate points in two or more stereo images. The NCC algorithm is one of the algorithms amongst correlation-type algorithms and is used for measuring similarity [31]. Finding the corresponding points by using NCC is complex. The corresponding points are assumed to possess the same brightness value in the reference and target patches. Theoretically, all points in the other image can be considered possible candidates. Finding the corresponding points is an ill-posed problem because depending on the brightness value only is an insufficient factor that can be used to measure similarity between images [31].

The NCC algorithm is based on fixing a template in the reference (left image) and moving a template on the target (right image), as shown in Figure 1. The NCC deformation, such as rotation and scale, cannot be adopted because it allows for two shift parameters between the reference and target patches.

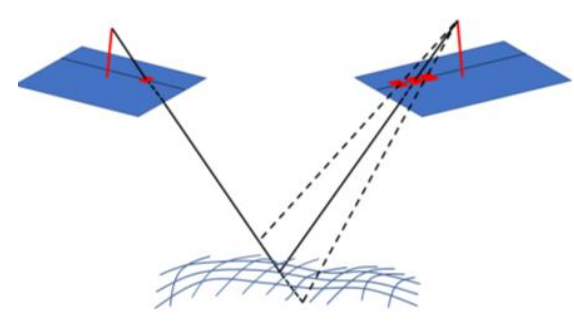

Figure 1. Process of image matching on two aerial images by using NCC.

In photogrammetry, NCC coefficient is used in image matching by statistically measuring similarity between the patches in the reference and the target image to find the corresponding points. This technique is based on measuring the radiometric values of pixels. The process is achieved by finding similarity using the patch in the reference and the target images with the size of template. The equation used to find similarity is

$$
N C C=\frac{\sigma_{g_{r} B_{S}}}{\sigma_{g_{r}} \cdot \sigma_{g_{S}}}=\frac{\sum_{i=1}^{m} \sum_{j=1}^{n}\left[\left(g_{T_{i, j}}-\bar{g}_{T}\right) \cdot\left(g_{S_{i, j}}-\bar{g}_{S}\right)\right]}{\sqrt{\sum_{i=1}^{m} \sum_{j=1}^{n}\left(g_{T_{i, j}}-\bar{g}_{T}\right)^{2}} \cdot \sqrt{\sum_{i=1}^{m} \sum_{j=1}^{n}\left(g_{S_{i, j}}-\bar{g}_{S}\right)^{2}}}
$$

Where $\sigma_{g T g S}$ is the covariance of the target and reference templates, $\sigma_{g T}$ is the standard deviation of the target template, and $\sigma_{g s}$ is the standard deviation of the reference template.

To find the corresponding point using NCC, the values of the NCC coefficient are firstly determined at each location. Then, the values that are greater than the threshold values are registered, and those lower than the threshold values are neglected. Lastly, the corresponding value is obtained by maximising the registered values. The threshold value should be selected cautiously. If the threshold value is extremely low, then the false point will be identified as a corresponding point although the maximum NCC is selected. The reason is that the template of the reference image will be matched with that of the target image at an incorrect location owing to low variance in both patches. If the threshold value is set to be extremely high, then few points will be selected as corresponding points because of considerable differences in the geometry and scale in images. Owing to the simplicity of using NCC, it is highly popular. NCC is robust at the location of low frequency and sufficient signals in terms of preserving minimum geometrical and radiometric distortion. However, aerial images do not possess these conditions [12]. The size of template exerts a significant effect on the value of NCC. Debella and Kaab [7] showed that the value of NCC fluctuates with the increase in the size of window. The value of NCC increases when the size of window enlarges, thereby leading to signals predominating over noise. Thus, noise is suppressed. Furthermore, the value of NCC decreases when the size of window decreased. 
The reason is that a large number of geometric features are introduced by introducing pixels of geometric distortions. In stereo images, the rotation is solved using the search along an epipolar line. The remaining problem is the distortion in geometrical image capturing. This problem can be solved using a large size of windows by including a large range of textures and a large number of features, although increasing the size of window will lead to identifying correct corresponding points [31]. Theoretically and in ideal cases, the maximum value of correlation is represented by the corresponding points. However, NCC is extremely sensitive to the location of occlusion and the changes in orientation and scale and results in deteriorated correlation [48].

\section{Probability Products of Normalised Cross Correlation (PPNCC)}

In the case of small template (e.g., 7×7), the small features around the pixel are recorded and the NCC image matching works successfully, as in the case of the roof of the slab and the ground in Figure 2 (template A). However, in the case of the edge or the parapet in Figure 2 (template B), the correlation is very low, thereby resulting in high probability of producing mismatch. However, this is not always the case. As shown in Figure 2 (template C), the size of window is increased. Given the different locations of camera and owing to occlusion, pixels of different features will disappear or be introduced in the object, thereby decreasing the value of NCC or increasing the value of NCC at different locations, respectively. This condition will produce outliers in the object.

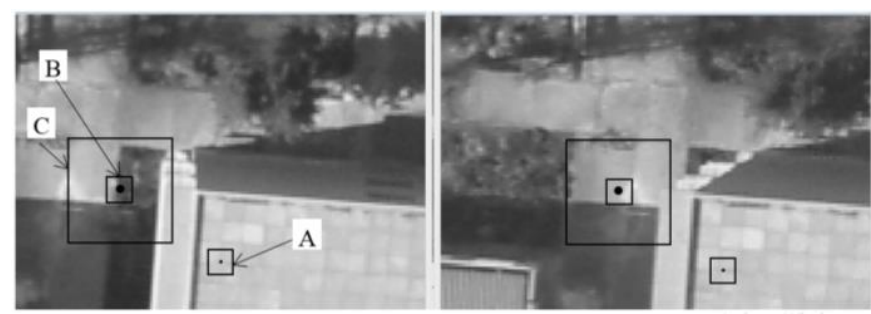

Figure 2. Stereo images that show the effect of the location of window and the change in the size of window.

In this research, the mismatch problem is solved by considering the probability of NCC by using different sizes of template. Then, the total probability of the NCC coefficients is determined. The algorithm is based on finding the products of probability of the crosscorrelation at each location, as shown in Equation (5).

$$
\begin{aligned}
P P N C C= & \prod_{k=m}^{k=k+2, n} P\left(W_{k \times k}\right)= \\
& P\left(W_{7 \times 7}\right) \cdot P\left(W_{9 \times 9}\right) \cdot P\left(W_{11 \times 11}\right) \cdot P\left(W_{n \cdot n}\right)
\end{aligned}
$$

Where $\mathrm{k}$ represents the size of window and it starts from the $7 \times 7$ template that is represented by $m \times m$. The product of window size continues until the size of window is $n \times n$. The probability is determined using
NCC divided by the summation of the NCC along the epipolar line, as shown in Equation (6).

$$
P\left(W_{k \times k}\right)_{i}=N C C_{i} / \sum_{i=0}^{l} N C C
$$

Where $i$ is the value of the probability at a specific pixel and 1 is the number of the pixels along the epipolar line.

\subsection{Independent Probability Values}

The determined quantity of NCC is an absolute value. Thus, for combining the values, they must be transformed into probability values to find the total probability values. The value range of NCC is represented between $[-1,1]$. If the correlation is 1 , then the reference and target templates are completely identical; if the correlation is equal to -1 , then the reference and opposite of the target are completely identical. Given that the focus is only on the object that is completely identical, the values less than 0 , which are considered negative, are neglected. The corresponding values of NCC at each size of window are not related to one another. For example, the corresponding point that is obtained using a window size of $7 \times 7$ is independent of the corresponding point that is obtained from the window size of $9 \times 9$. The same situation is applied to other sizes of window because the obtained corresponding points from each size of window are considered independent events and are thus based on probability rule, total events that occur when the probability values are obtained by multiplying events by each other (Equation (7)) [28]. For more than two windows, the total probability is obtained by multiplying each of them by each other because the values are independent.

$$
P\left(W_{7 \times 7} \cdot W_{9 \times 9}\right)=P\left(W_{7 \times 7}\right) \cdot P\left(W_{9 \times 9}\right)
$$

The conversion of the threshold value from absolute value to probability value is shown in Equation (8). The value is converted into probability value by dividing the threshold value (e.g., 0.8) over the multiplication of the summation value of NCC for all window sizes used in the algorithm. The threshold value that is in the PPNCC algorithm is called the threshold-probability value to distinguish it from the threshold that is used in the NCC coefficient.

$$
\begin{aligned}
& \text { Threshold }_{\text {probability }} \\
& =\frac{\text { Value of the threshold (e.g.0.8) }}{\sum_{0}^{n} N C C_{7 \times 7} \cdot \sum_{0}^{n} N C C_{9 \times 9} \cdot \sum_{0}^{n} N C C_{11 \times 11}}
\end{aligned}
$$

\subsection{Summary of the PPNCC Algorithm}

The proposed algorithm is based on finding the correlation by using various sizes of window (e.g., $7 \times 7, \quad 9 \times 9, \quad 15 \times 15, \quad 17 \times 17,19 \times 19 \ldots)$. Then, the probability values of each pixel are calculated. Thereafter, the total probability is determined by 
finding the product of the probability values. The corresponding point is determined by selecting the maximum of total probability. The results show that the proposed algorithm can successfully identify the corresponding points. The drawback of this method is the extensiveness of the consumed time because, at each specific point, the value of the cross-correlation is determined and the probability is calculated by using various sizes of window. Furthermore, large size of window will consume high time of computation.

\section{Experiment Result and Analysis}

In this section, the algorithm is applied and compared with NCC. Firstly, the algorithm is applied to a set of points that is specified to be complex, and it is counted to be four points. Then, the algorithm is applied to a group of points that are obtained from the Harris interest point operator.

\subsection{Study Area and Test Points}

The study area is located in the college of the engineering campus. The digital aerial image is acquired with UltraCAM Xp camera (2012), with a focal length of $100.5 \mathrm{~mm}$. The images are available in the surveying engineering department for educational and research purposes. For aero triangulation, a set of ground control points is measured at the site using static GPS. To obtain exterior parameters of images, summit evolution software is used, which is later used to determine the 3D coordinates of points by using collinearity equations. The images are specified to be multispectral (three bands) with a size of 11310 rows by 17310 columns. For the experimental test, the grey value is extracted using OpenCV tool. The geodetic datum of the GCPs is located in the WGS84-38N zone and the vertical datum is referred to WGS84 ellipsoidal datum.

For the algorithm implementation, a sample of the site is extracted to accelerate the operation. The lower left coordinate of the selected sample is $5500 \times 6350$ pixels and that of the upper right is $6500 \times 7100$ pixels, with GSD of the image measured to be $0.132 \mathrm{~m} 2$ pixels. Thus, the total area comprises 750,000 pixels. The size of the study area is $99 \mathrm{~m}$ high by $131 \mathrm{~m}$ wide.

In photogrammetry, applying image matching on every pixel is a time-consuming operation. To solve this problem, only a few pixels are selected and specified as interest features. The matching is applied to interest points instead of applying the operation to all the pixels. These interest points are the same points that the operator selects to find the coordinates, such as corners of a road or buildings and distinct points. Different algorithms have been implemented in photogrammetry for interest point extraction such as foerstner and morveico [25], Harris, SUSAN, SURF and FAST [8]. In the current research, the Harris operator is implemented because the code is available in the OpenCV environment [10]. The Harris operator has been implemented to detect interest points [15]. The Harris detector is specified to extract corners only, thereby leading to discontinuity of the object and surface [14]. As shown in Figure 3, the Harris operator successfully identifies the corner of the building. The location is of high contrast. The number of detected points is 2680 amongst the 750,000 pixels. These points provide high correlation because they are located at the edge of the building and possess high SNR.
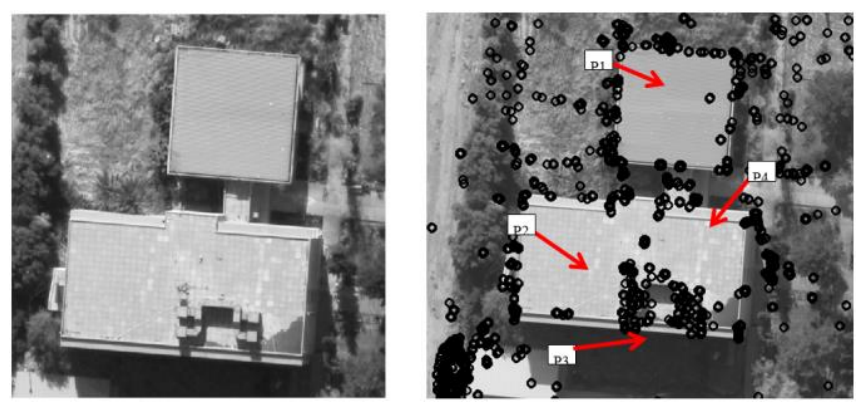

Figure 3. Test area used in the application: the left image represents the test area, and the right image represents the interest point obtained using the Harris operator and the four points used in the evaluation.

For a particular point evaluation of the proposed technique, four points are identified firstly for testing the algorithms (Figure 4). Then, the algorithm is applied to the test area. The four points are located at different locations: $\mathrm{P} 1$ is located on area of homogenous texture, as shown in Figure 4-a. P2 is located on the edge of the building of large variations in texture, as shown in Figure 4-b. P3 is located in a shadow area of very low value of SNR, as shown in Figure 4-c. The fourth point P4 is positioned on the surface of the wall of a highly complex location because of high geometric distortion, as shown in Figure 4-d. P1 and P2 are obtained from the Harris point operator and are specified to be corner points of extremely high variance amongst the surrounding points. $\mathrm{P} 3$ and $\mathrm{P} 4$ are located arbitrarily in shadow and flat surfaces of small variance in texture. The four locations are considered critical in the matching.

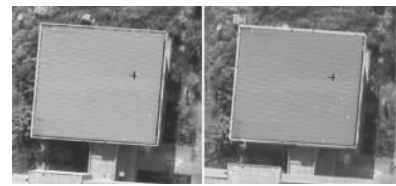

a) Location of $\mathrm{P} 1$ is identified on flat roof.

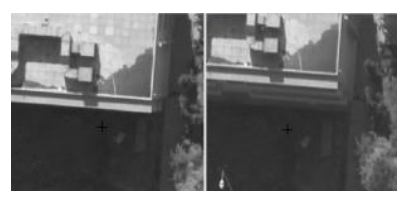

c) Is located in the shadow area.

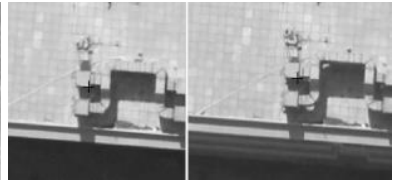

b) Location of P2 is located on the roof near mechanical structure.

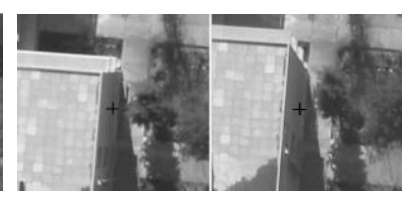

d) Is located in the complex area on the surface of the building.
Figure 4. Points that are used in the evaluation. 


\subsection{Testing NCC}

As was stated in literature and as tested in this section, NCC identifies most of the points successfully. However, some of the points are not identified correctly, especially at the location of the flat area or low feature area (Figure 4 (a, c and d)). This failure is due to the lack of significant variance in the surrounding pixels. These mismatched points are considered outliers that are difficult to be identified automatically at a later stage, thereby causing errors in the model especially in the case of obtaining highquality DSM [18]. The value of the correlation need not be constant with the image pixels. This correlation depends on the brightness of the pixels. However, increasing the size of window, such as from $7 \times 7$ to $13 \times 13$ with a threshold correlation of 0.5 , improves the result (e.g., mismatches are decreased), but some mismatches remain. In the window size of $13 \times 13$, when the threshold value is changed from 0.5 to 0.9 , some of the points are identified as mismatched but in a small amount (Figure 5 ( $a$ and $b$ )). The increase in the threshold value of the NCC coefficient exerts an adverse effect on the result, in which the number of extracted points is decreased dramatically. Thus, the shape of the buildings will not be identified correctly.

The maximum value of the correlation is not necessarily the candidate point. High correlation usually does not represent the maximum correlation. The results indicate that no specific criteria are available for selecting the candidate point on the basis of the threshold value or the window size. Therefore, using NCC to find the corresponding point is considered an ill-posed problem.

In the evaluation, firstly, the test is started by using NCC to find the best size of window that can successfully identify the corresponding points. In the NCC test, 15 different sizes of window (from $7 \times 7$ to $35 \times 35$ ) are used. The NCC algorithm is applied to the four identified points, as shown in Figures 3 and 4. The used window is in an odd number; thus, the interest point is located at the centre of the window or kernel. The results are individually shown in Figure 6. For each point of corresponding size of window, the maximum correlation and the correlation at the corresponding location are identified.

The result for point $\mathrm{P} 1$ shows that the corresponding point is identified correctly with all window sizes from $9 \times 9$ to $35 \times 35$, except for the window size of $7 \times 7$. The curve shows a steady increase, thereby indicating that the pixels are positioned in the area of low geometry distortion (e.g., minimum relief area). Thus, NCC can accurately specify the corresponding points. Point $\mathrm{P} 2$ is located on the edge of the mechanical object that possesses various geometric features. Similarly, the value of the maximum NCC is steady and is higher than 0.9 . However, the problem beyond the window size of $15 \times 15$ is that the matching fails to locate the correct corresponding points. The reason is that various pixels exist in the window. As regards points $\mathrm{P} 3$ and $\mathrm{P} 4$, they are identified as arbitrary with no boundary feature around them and the points are of very low value of SNR. The result of the point is not steady, and the curve fluctuates, especially with large window sizes of $33 \times 33$ and $25 \times 25$ because of the sudden introduction of different pixels in the window. The NCC algorithm acts effectively and attains highly accurate results in the case of the flat surface with no relief in the window region and possesses different geometric features that increase the value of SNR. With NCC, no specific window can be used. Thus, using a specific window for image matching that can successfully identify the corresponding point at all the pixels is impossible.

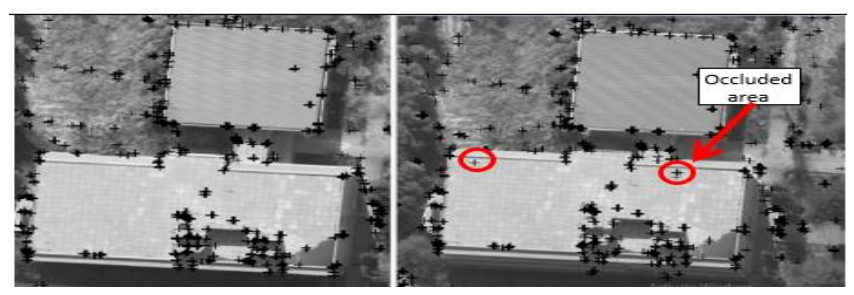

a) Window size of $13 \times 13$ with a correlation threshold of 0.5 .

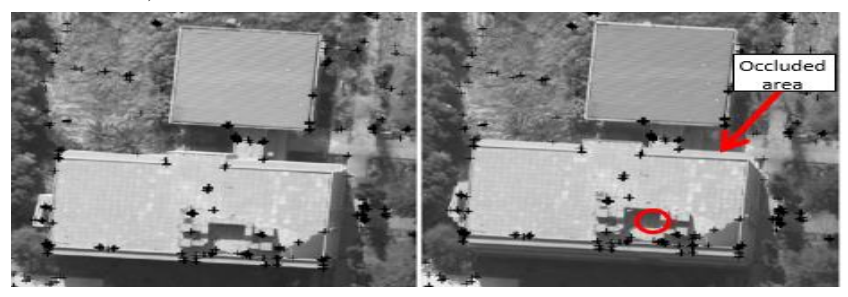

b) Window size of $13 \times 13$ with a correlation threshold of 0.9 .

Figure 5. Using NCC to find the corresponding points. Red circles show the mismatches.

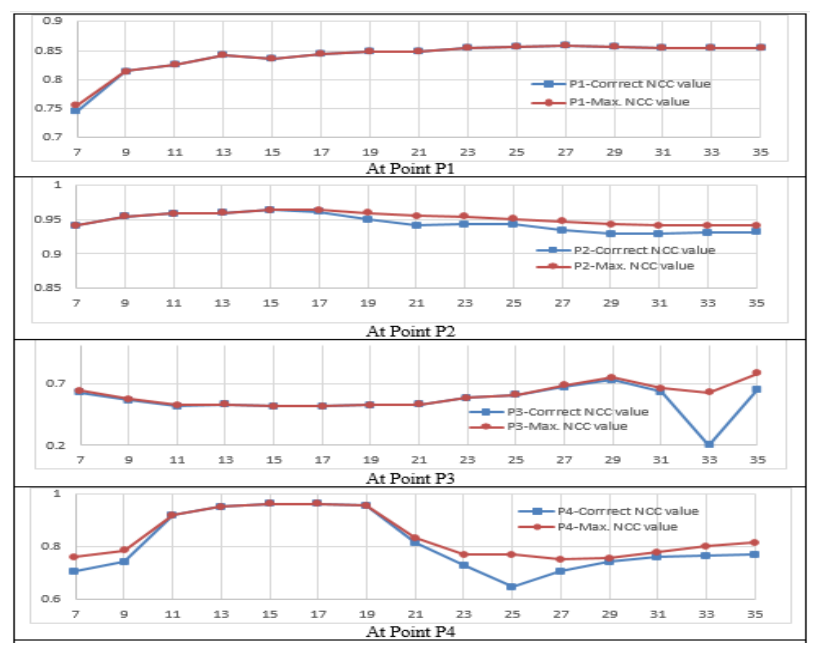

Figure 6. Comparison between maximum values of NCC for the selected test points, P1, P2, P3 and P4, and the value of the NCC at the corresponding point.

The NCC technique is also applied to the study area (Figure 3) by using the interest points that are determined using the Harris operator. Different sizes of window are used with NCC coefficient determination. As shown in Table 1, the number of detected points decreases with the increase in the size 
of window because the number of pixels with a variant value increases, thereby leading to variance values in the used windows. This process decreases the correlation value. Table 1 clearly indicates that outliers are detected in all different sizes of window. No specific size of window can be used with NCC. Thus, no robust size of window can be used even when the value of threshold is increased. Nevertheless, increasing the size of window will not necessarily eliminate the outliers.

Table 1. Applying NCC on 3233 Harris points at the test area implementing different sizes of window, +ve outlier exist, -ve there is no outlier.

\begin{tabular}{|c|c|c|c|c|}
\hline $\begin{array}{l}\text { Correlation } \\
\text { window size }\end{array}$ & $\begin{array}{c}\text { Correlation } \\
\text { threshold }\end{array}$ & $\begin{array}{l}\text { Detected } \\
\text { points }\end{array}$ & $\%$ detected & Outlier existing status \\
\hline $7 \times 7$ & \multirow{7}{*}{0.8} & 2825 & $87.5 \%$ & $+\mathrm{ve}$ \\
\hline $9 \times 9$ & & 2707 & $83.7 \%$ & $+\mathrm{ve}$ \\
\hline $11 \times 11$ & & 2642 & $81.7 \%$ & $+\mathrm{ve}$ \\
\hline $13 \times 13$ & & 2577 & $79.7 \%$ & $+\mathrm{ve}$ \\
\hline $15 \times 15$ & & 2567 & $79.4 \%$ & $+\mathrm{ve}$ \\
\hline $21 \times 21$ & & 2603 & $80.5 \%$ & $+\mathrm{ve}$ \\
\hline $25 \times 25$ & & 2590 & $80.1 \%$ & $+\mathrm{ve}$ \\
\hline $7 \times 7$ & \multirow{7}{*}{0.9} & 2225 & $68.8 \%$ & $+\mathrm{ve}$ \\
\hline $9 \times 9$ & & 2171 & $67.2 \%$ & $-\mathrm{ve}$ \\
\hline $11 \times 11$ & & 2148 & $66.4 \%$ & $+\mathrm{ve}$ \\
\hline $13 \times 13$ & & 2108 & $65.2 \%$ & $-\mathrm{ve}$ \\
\hline $15 \times 15$ & & 2072 & $64.1 \%$ & $-\mathrm{ve}$ \\
\hline $21 \times 21$ & & 2030 & $62.8 \%$ & $+\mathrm{ve}$ \\
\hline $25 \times 25$ & & 1921 & $59.4 \%$ & $+\mathrm{ve}$ \\
\hline
\end{tabular}

In the outlier inspection, the object's 3D XYZ coordinates are determined using collinearity equation. This process is achieved on the basis of the obtained image coordinate. This corresponding illustration is shown in Figure 7. The 3D coordinates are visualized using cloud compare software, thus the outlier is detected visually. Although a high percentage of the points are detected, some of them are considered outliers, thereby leading to erroneous matching. Thus, false points are produced in the DSM, as shown in Figure 7.

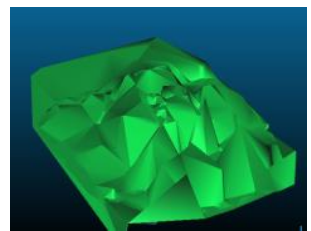

a) Produced DSM with a window size of $13 \times 13$ and the produced outliers

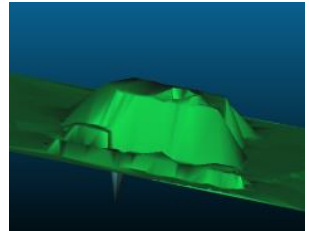

b) Detected outliers that appear as a spike at the bottom of the surface by using a window size of $25 \times 25$.
Figure 7. Generated DSM using NCC with a threshold value of 0.8 .

\subsection{Testing PPNCC}

In this section, the proposed PPNCC algorithm is implemented. The first trial is started using window sizes of $7 \times 7$ and $9 \times 9$. Thereafter, larger sizes of window are used for each trial. The values of threshold that are applied in the PPNCC algorithm are based on the value of probability but are equivalent to the same threshold values of NCC. In the testing, the PPNCC algorithm is applied to the four points are stated in Figure 4 . Firstly, the probability of window sizes $7 \times 7$ and $9 \times 9$ are determined. Then, the window size is increased progressively to identify the difference. The matching result is listed in Table 2. The analysis shows that the same probability value of 0.8 used in NCC does not work with PPNCC at all the four points, except for point $\mathrm{P} 2$, and with window sizes from $7 \times 7$ to $9 \times 9$, from $7 \times 7$ to $11 \times 11$ and from $7 \times 7$ to $13 \times 13$. Skipped to window sizes from $7 \times 7$ to $21 \times 21$ and from $7 \times 7$ to $25 \times 25$ to save time. Notably, little difference in the result is found using intermediate sizes of window. The used threshold value is very high, and no matching points are identified; the result is nil.

When the threshold value is lowered, a large number of corresponding points are identified at all selected locations, except at point 3 , which fails with large sizes of window unlike in other sizes of window. The reason is that the point is located in a shadow area of very low value of SNR and very low correlation, thereby resulting in very small total probability.

Table 2. Using PPNCC for image matching implementing different sizes of window with a threshold value of 0.8 ; "nil" indicates that no matching appears because of high threshold value (+ve means point are correctly identified).

\begin{tabular}{|c|c|c|c|c|c|}
\hline Correlation window size & \begin{tabular}{|c|} 
Threshold- \\
probability value
\end{tabular} & $\begin{array}{c}\text { Point } \\
\text { p1 }\end{array}$ & Point p2 & $\begin{array}{c}\text { Point } \\
\text { p3 }\end{array}$ & Point p4 \\
\hline $7 \times 7,9 \times 9$ & \multirow{6}{*}{ p } & nil & $+\mathrm{ve}$ & nil & nil \\
\hline $7 \times 7,9 \times 9,11 \times 11$ & & nil & $+\mathrm{ve}$ & nil & nil \\
\hline $7 \times 7,9 \times 9,11 \times 11,13 \times 13$ & & nil & $+\mathrm{ve}$ & nil & nil \\
\hline $\begin{array}{c}7 \times 7,9 \times 9,11 \times 11,13 \times 13, \\
15 \times 15\end{array}$ & & nil & nil & nil & nil \\
\hline $\begin{array}{c}7 \times 7,9 \times 9,11 \times 11,13 \times 13, \\
15 \times 15,17 \times 17,19 \times 19,21 \times 21\end{array}$ & & nil & nil & nil & nil \\
\hline $\begin{array}{c}7 \times 7,9 \times 9,11 \times 11,13 \times 13, \\
15 \times 15,17 \times 17,19 \times 19,21 \times 21, \\
23 \times 23,25 \times 25\end{array}$ & & nil & nil & nil & nil \\
\hline $7 \times 7,9 \times 9$ & \multirow{6}{*}{0.4} & $+\mathrm{ve}$ & $+\mathrm{ve}$ & nil & $+\mathrm{ve}$ \\
\hline $7 \times 7,9 \times 9,11 \times 11$ & & $+\mathrm{ve}$ & $+\mathrm{ve}$ & nil & $+\mathrm{ve}$ \\
\hline $7 \times 7,9 \times 9,11 \times 11,13 \times 13$ & & $+\mathrm{ve}$ & $+\mathrm{ve}$ & nil & $+\mathrm{ve}$ \\
\hline $\begin{array}{c}7 \times 7,9 \times 9,11 \times 11,13 \times 13, \\
15 \times 15\end{array}$ & & nil & $+\mathrm{ve}$ & nil & $+\mathrm{ve}$ \\
\hline $\begin{array}{c}7 \times 7,9 \times 9,11 \times 11,13 \times 13, \\
15 \times 15,17 \times 17,19 \times 19,21 \times 21\end{array}$ & & nil & $+\mathrm{ve}$ & nil & \\
\hline $\begin{array}{c}7 \times 7,9 \times 9,11 \times 11,13 \times 13, \\
15 \times 15,17 \times 17,19 \times 19,21 \times 21, \\
23 \times 23,25 \times 25\end{array}$ & & nil & $+\mathrm{ve}$ & nil & nil \\
\hline $7 \times 7,9 \times 9$ & \multirow{6}{*}{0.1} & $+\mathrm{ve}$ & $+\mathrm{ve}$ & $+\mathrm{ve}$ & $+\mathrm{ve}$ \\
\hline $7 \times 7,9 \times 9,11 \times 11$ & & $+\mathrm{ve}$ & $+\mathrm{ve}$ & $+\mathrm{ve}$ & $+\mathrm{ve}$ \\
\hline $7 \times 7,9 \times 9,11 \times 11,13 \times 13$ & & $+\mathrm{ve}$ & $+\mathrm{ve}$ & nil & $+\mathrm{ve}$ \\
\hline $\begin{array}{c}7 \times 7,9 \times 9,11 \times 11,13 \times 13, \\
15 \times 15\end{array}$ & & $+\mathrm{ve}$ & $+\mathrm{ve}$ & nil & $+\mathrm{ve}$ \\
\hline $\begin{array}{c}7 \times 7,9 \times 9,11 \times 11,13 \times 13, \\
15 \times 15,17 \times 17,19 \times 19,21 \times 21\end{array}$ & & $+\mathrm{ve}$ & $+\mathrm{ve}$ & nil & $+\mathrm{ve}$ \\
\hline $\begin{array}{c}7 \times 7,9 \times 9,11 \times 11,13 \times 13, \\
15 \times 15,17 \times 17,19 \times 19,21 \times 21, \\
23 \times 23,25 \times 25\end{array}$ & & $+\mathrm{ve}$ & $+\mathrm{ve}$ & nil & $+\mathrm{ve}$ \\
\hline
\end{tabular}

The algorithm is also applied to the study area using interest points from the Harris point detector. Table 3 shows that PPNCC with window sizes from $7 \times 7$ to $9 \times 9$ and a threshold-probability value of 0.9 presents no outliers. The outliers vanish when the threshold value is lowered by one digit, such as when the threshold values are 0.8 and 0.2 with window sizes from $7 \times 7$ to $13 \times 13$ and from $7 \times 7$ to $25 \times 25$, respectively. Notably, as the sizes of window enlarge and the threshold-probability value decreases, a large 
number of points are detected because a high matching probability is introduced in the overall windows. This process leads to decreasing the probability of false matching. When the window size is from $7 \times 7$ to $9 \times 9$, the percentage of detected points is $46.2 \%$ with a threshold-probability value of 0.9 , whereas detection increases gradually to $71.5 \%$ with window sizes from $7 \times 7$ to $25 \times 25$ using a threshold-probability value of 0.2 . As shown in Table 1, detecting correct points with a specific threshold value is intermittent for NCC; this is due to variant geometric and radiometric distortion introduced with each window size. A specific threshold value is also not guaranteed to eliminate outliers. For PPNCC, the probability of detecting the exact corresponding points increases with the number of windows. Moreover, the number of points increases. However, when the number of windows detecting points increases, the processing time increases as well. The reason for obtaining a high rate of corresponding points is due to integrating the probability of different window sizes. Even in the case if one or more window sizes are failed, because of varying geometric and radiometric distortions, when integrating different window sizes will overcome the failed window size thus the probability of selecting the correct corresponding point is increased. Table 3 indicates that the optimum window sizes are from $7 \times 7$ to $15 \times 15$ with a threshold-probability value of 0.7 . Window sizes from $7 \times 7$ to $25 \times 25$ with a threshold-probability value of 0.2 can eliminate outliers. However, this task requires much time to process.

Table 3. Using PPNCC for matching 3233 Harris interest points with different sizes of window, +ve outlier exist, -ve there is no outlier.

\begin{tabular}{|c|c|c|c|c|}
\hline Correlation window size & \begin{tabular}{|c|} 
Total \\
Probability- \\
threshold
\end{tabular} & \begin{tabular}{|c|}
$\begin{array}{c}\text { Detected points } \\
\text { out of } 3233 \\
\text { points }\end{array}$ \\
\end{tabular} & $\%$ detected & $\begin{array}{c}\text { Outliers } \\
\text { exist }\end{array}$ \\
\hline \multirow{4}{*}{$7 \times 7,9 \times 9$} & 0.6 & 2827 & $87.7 \%$ & $+\mathrm{ve}$ \\
\hline & 0.7 & 2594 & $80.2 \%$ & $+\mathrm{ve}$ \\
\hline & 0.8 & 2223 & $68.8 \%$ & $+\mathrm{ve}$ \\
\hline & 0.9 & 1494 & $46.2 \%$ & -ve \\
\hline \multirow{5}{*}{$7 \times 7,9 \times 9,11 \times 11$} & 0.4 & 2860 & $88.5 \%$ & $+\mathrm{ve}$ \\
\hline & 0.5 & 2699 & $83.5 \%$ & $+\mathrm{ve}$ \\
\hline & 0.6 & 2486 & $76.9 \%$ & $+\mathrm{ve}$ \\
\hline & 0.7 & 2235 & $69.1 \%$ & $+\mathrm{ve}$ \\
\hline & 0.8 & 1853 & $57.3 \%$ & -ve \\
\hline \multirow{6}{*}{$7 \times 7,9 \times 9,11 \times 11,13 \times 13$} & 0.3 & 2807 & $86.8 \%$ & $+\mathrm{ve}$ \\
\hline & 0.4 & 2652 & $82.0 \%$ & $+\mathrm{ve}$ \\
\hline & 0.5 & 2455 & $75.9 \%$ & $+\mathrm{ve}$ \\
\hline & 0.6 & 2259 & $69.9 \%$ & $+\mathrm{ve}$ \\
\hline & 0.7 & 1981 & $61.3 \%$ & $-\mathrm{ve}$ \\
\hline & 0.8 & 1554 & $48.1 \%$ & $-\mathrm{ve}$ \\
\hline \multirow{7}{*}{$\frac{7 \times 7,9 \times 9,11 \times 11,13 \times 13}{15 \times 15}$} & 0.2 & 2811 & $86.9 \%$ & $+\mathrm{ve}$ \\
\hline & 0.3 & 2656 & $82.2 \%$ & $+\mathrm{ve}$ \\
\hline & 0.4 & 2460 & $76.1 \%$ & $+\mathrm{ve}$ \\
\hline & 0.5 & 2294 & $71.0 \%$ & $+\mathrm{ve}$ \\
\hline & 0.6 & 2107 & $65.2 \%$ & $+\mathrm{ve}$ \\
\hline & 0.7 & 1771 & $54.8 \%$ & $-\mathrm{ve}$ \\
\hline & 0.8 & 1274 & $39.4 \%$ & $-\mathrm{ve}$ \\
\hline \multirow{3}{*}{$\begin{array}{c}7 \times 7,9 \times 9,11 \times 11,13 \times 13 \\
15 \times 15,17 \times 17,19 \times 19 \\
21 \times 21\end{array}$} & 0.2 & 2479 & $76.7 \%$ & $+\mathrm{ve}$ \\
\hline & 0.3 & 2280 & $70.5 \%$ & $+\mathrm{ve}$ \\
\hline & 0.4 & 2138 & $66.1 \%$ & $-\mathrm{ve}$ \\
\hline \multirow{4}{*}{$\begin{array}{c}7 \times 7,9 \times 9,11 \times 11,13 \times 13 \\
15 \times 15,17 \times 17,19 \times 19 \\
21 \times 21,23 \times 23,25 \times 25\end{array}$} & 0.1 & 2525 & $78.1 \%$ & $+\mathrm{ve}$ \\
\hline & 0.2 & 2310 & $71.5 \%$ & $-\mathrm{ve}$ \\
\hline & 0.3 & 2144 & $66.3 \%$ & $-\mathrm{ve}$ \\
\hline & 0.4 & 1953 & $60.4 \%$ & $-\mathrm{ve}$ \\
\hline
\end{tabular}

The produced DSMs for window sizes from $7 \times 7$ to $15 \times 15$ with a threshold value of 0.7 and from $7 \times 7$ to $21 \times 21$ with a threshold value of 0.4 in Figure 8 -a and b) indicates no significant difference and nearly similar detected points. Thus, PPNCC with small size of window is preferred to incur less processing time.
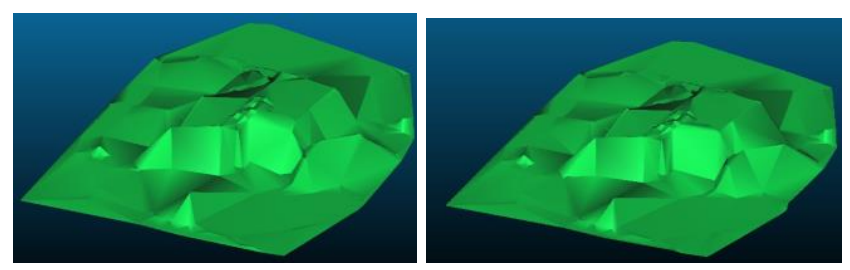

a) With window sizes from $7 \times 7$ to $15 \times 15$ b) With window sizes from $7 \times 7$ to $21 \times 21$ by using a threshold-probability value of by using a threshold-probability value of 0.7 . 0.4 .

Figure 8. Generated DSM using PPNCC.

Increasing the value of threshold-probability in the PPNCC algorithm has the advantages of removing outliers. However, some of the Harris interest points are vanished from the tested area, especially at the corner of the building (Figure 9). Thus, the shape of the building is deformed, thereby producing an approximate shape of the building (Figure 8).
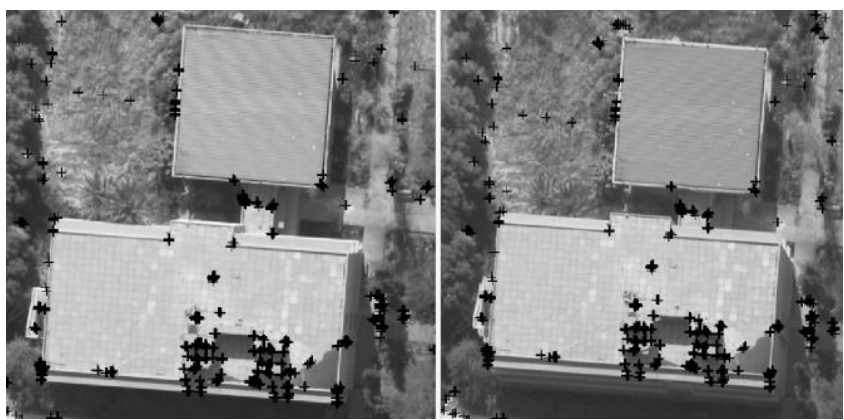

Figure 9. Using high value of threshold-probability to avoid outliers. Some of the Harris points of interest are removed in the corner of the building, thereby causing the shape of the building to not be truly identified. The cross marks represent the matched points.

The algorithm is applied to another test area, as shown in Figure 10. The area covered is 750 rows by 1100 columns. The results are shown in Figure 10 -b), which indicates that no outliers exist in the model. Thus, the 3D model is successfully generated. The total number of the Harris points is 9226 . The total number of detected points is 2196 .
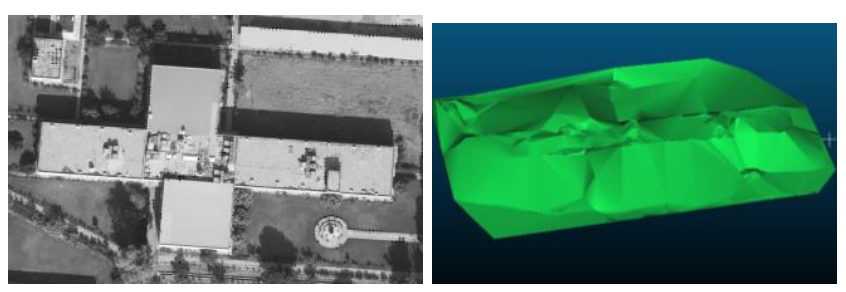

Figure 10. Generated DSM on another test area: (a) second test area that is used in the application and (b) PPNCC using window sizes from $7 \times 7$ to $15 \times 15$ with threshold-probability values of 0.7 


\section{Conclusions}

This paper presents a method for image matching called PPNCC, which is based on the NCC technique. In NCC, no specific size of window can be used to detect the matching points. At some locations, certain sizes of window can find the corresponding points whilst other sizes of window need to be used to find the corresponding points at other points. In this research, combining different sizes of window is possible by finding the probability of each window and integrating the windows to find the correct corresponding point. The results show that, by integrating large sizes of window in the algorithm, the probability of locating the corresponding points increases. Thus, the mismatch is decreased.

A few researchers have claimed that, if the operator cannot detect the corresponding point, then the algorithm cannot achieve the matching [36]. However, the PPNCC algorithm can detect matching points even at problematic locations, such as flat smooth surface with no feature and of extremely low value of SNR. In the qualitative assessment, the cloud compare software is used to detect outliers.

The results of PPNCC are compared with those of NCC. The number of detected points is higher with PPNCC than with NCC, and PPNCC can find the corresponding points more robustly than does NCC owing to integrating a large number of windows. The probability of detecting the corresponding points increases when a large number of windows are used. In this way, the processing time increases. For NCC, outliers still exist on the Harris points of high value of SNR even when large size of window is used.

\section{References}

[1] An R., Gong P., Wang H., Feng X., Xiao P., Chen Q., Zhang Q., Chen C., and Yan P., "A Modified PSO Algorithm for Remote Sensing Image Template Matching," Photogrammetric Engineering and Remote Sensing, vol. 76, no. 4, pp. 379-389, 2010.

[2] Blum R. and Liu Z., Multi-Sensor Image Fusion and its Applications, CRC Press, 2005.

[3] Burger W. and Burge M., Principles of Digital Image Processing, Springer, 2013.

[4] Chen L., Lee L., and Lee S., "DTM Generation Using SPOT Digital Data," in Proceedings of ISPRS Commission III, Kyoto, pp. 100-109, 1988.

[5] Chen Y., Tseng Y., Hsieh C., Wang P., and Tsai P., "Object-Space Multi-Image Matching of Mobile-Mapping Image Sequences," in Proceedings of International Archives of the Photogrammetry, Remote Sensing and Spatial Information Sciences, Melbourne, pp. 465-470, 2012.
[6] Dall'Asta E. and Roncella R., "A Comparison of Semiglobal and Local Dense Matching Algorithms for Surface Reconstruction," in Proceedings of International Archives of the Photogrammetry, Remote Sensing and Spatial Information Sciences, Riva del Garda, pp. 187194, 2014.

[7] Debella-Gilo M. and Kaab A., "Locally Adaptive Template Sizes for Matching Repeat Images of Earth Surface Mass Movements," ISPRS Journal of Photogrammetry and Remote Sensing, vol. 69, pp. 10-28, 2012.

[8] Detchev I., Habib A., and Rau J., "Image Matching for 3D Photogrammetric Reconstruction," in Proceedings of the $32^{\text {nd }}$ Asian Conference on Remote Sensing, Taipei, pp. 247-252, 2011.

[9] Di-Rita M., Nascetti A., and Crespi M., "Open Source Tool for Dsms Generation from High Resolution Optical Satellite Imagery: Development and Testing of an OSSIM Plugin," International Journal of Remote Sensing, vol. 38, no. 7, pp. 1788-1808, 2017.

[10] Doxygen., "Harris Corner Detector," Available from:

http://docs.opencv.org/3.1.0/d4/d7d/tutorial_harr is_detector.html, Last Visited, 2018.

[11] Gesto-Diaz M., Tombari F., Gonzalez-Aguilera D., Lopez-Fernandez L., and RodriguezGonzalvez P., "Feature Matching Evaluation for Multimodal Correspondence," ISPRS Journal of Photogrammetry and Remote Sensing, vol. 129, pp. 179-188, 2017.

[12] Gruen A., "Adaptive Least Squares Correlation: A Powerful Image Matching Technique," South African Journal of Photogrammetry, Remote Sensing and Cartography, vol. 14, no. 3, pp. 175-187, 1985.

[13] Gruen A., "Development and Status of Image Matching in Photogrammetry," The Photogrammetric Record, vol. 27, no. 137, pp. 36-57, 2012.

[14] Guerrero M., "A Comparative Study of Three Image Matcing Algorithms: Sift, Surf, and Fast," MSc Thesis, Utah State University, 2011.

[15] Harris C. and Stephens M., "A Combined Corner and Edge Detector," in Proceedings of the $4^{\text {th }}$ Alvey Vision Conference, Manchester, pp. 147$151,1988$.

[16] Hashemi N., Aghdam R., Ghiasi A., and Fatemi P., "Template Matching Advances and Applications in Image Analysis," American Scientific Research Journal for Engineering, Technology, and Sciences, vol. 26, no. 3, 2016.

[17] Hirschmuller H., "Stereo Processing By Semiglobal Matching and Mutual Information," IEEE Transactions on Pattern Analysis and 
Machine Intelligence, vol. 30, no. 2, pp. 328-41, 2008.

[18] Höhle J. and Potuckova M., "Assessment of the Quality of Digital Terrain Models," European Spatial Data Research (EuroSDR), 2011.

[19] Hong G. and Zhang Y., "Wavelet-Based Image Registration Technique for High-Resolution Remote Sensing Images," Computers and Geosciences, vol. 34, no. 12, pp. 1708-1720, 2008.

[20] Jin X. and Davis C., "Automated Building Extraction from High-Resolution Satellite Imagery in Urban Areas Using Structural, Contextual, and Spectral Information," EURASIP Journal on Advances in Signal Processing, vol. 14, pp. 2196-2206, 2005.

[21] Joglekar J. and Gedam S., "Area Based Stereo Image Matching Technique Using Hausdorff Distance and Texture Analysis," in Proceedings of International Archives of the Photogrammetry, Remote Sensing and Spatial Information Sciences, Munich, pp. 109-114, 2011.

[22] Koh A., Schonermark M., and Theuwisse A., "Detectors and Sensors," in Manual of Photogrammetry, 2013.

[23] Kraus K., Photogrammetry: Geometry from Images and Laser Scans, De Gruyter, 2007.

[24] Mayer H., Sester M., and Vosselman G., "Basic Computer Vision Techniques," in Manual of Photogrammetry, 2013.

[25] Mikhail E., Bethel J., and McGlone J., Introduction to Modern Photogrammetry, Wiley, 2001.

[26] Oh J., "Novel Approach to Epipolar Resampling of HRSI and Satellite Stereo Imagery-based Georeferencing of Aerial Images," PhD Thesis, The Ohio State University, 2011.

[27] Oh J., Toth C., and Grejner-Brzezinska D., "Automatic Georeferencing of Aerial Images Using Stereo High-Resolution Satellite Images" Photogrammetric Engineering and Remote Sensing, vol. 77, no. 11, pp. 1157-1168, 2011.

[28] Papoulis, A. and Pillai, S., Probability, Random Variables, and Stochastic Processes, Tata McGraw-Hill Education, 2002.

[29] Qin X., Li L., and Tian S., "A New Image Matching Algorithm with Modified Greedy Algorithm For Remote Sensing Imagery," in Proceedings of ISPRS Workshop on Service and Application of Spatial Data Infrastructure. Hangzhou, pp. 113-120, 2003.

[30] Qiu C., Schmitt M., and Zhu X., "A Tie Point Matching Strategy For Very High Resolution SarOptical Stereogrammety over Urban Areas," in Proceedings of International Archives of the Photogrammetry, Remote Sensing and Spatial Information Sciences, Hannover, pp. 55-61, 2017.

[31] Roma N., Santos-Victor J., and Tomé J.,
Empirical Evaluation Methods in Computer Vision, World Scientific, 2002.

[32] Singh P. and Agarwal S., "A Region Adaptive Robust Watermarking Scheme Based on Homogeneity Analysis," The International Arab Journal of Information Technology, vol. 13, no. 5, pp. 517-522, 2016.

[33] Sirguey P., Cullen N., and Santos J., "The New Digital Orthometric Elevation Model of Kilimanjaro," in Proceedings of Research Locate, Canberra, pp. 108-117, 2014.

[34] Sonka M., Hlavac V., and Boyle R., Image Processing, Analysis, and Machine Vision, Cengage Learning, 2014.

[35] Tachikawa T., Kaku M., and Iwasaki A., "Aster GDEM Version 2 Validation Report," Report to the ASTER GDEM Version 2, University of Tokyo, 2011.

[36] Wang R., Ma J., Huang H., and Shi W., "Improved Nonsubsampled Contourlet Transform for Multi-Sensor Image Registration," Photogrammetric Engineering and Remote Sensing, vol. 79, no. 7, pp. 51-66 2013.

[37] Weeks A., Fundamentals of Electronic Image Processing, Wiley-IEEE Press, 1996.

[38] Wen G., Lv J., and Yu W., "A HighPerformance Feature-Matching Method for Image Registration By Combining Spatial And Similarity Information," IEEE Transactions on Geoscience and Remote Sensing, vol. 46, no. 4, pp. 1266-1277, 2008.

[39] Wolf P., DeWitt B., and Wilkinson B., Elements of Photogrammetry with Application in GIS, McGraw-Hill Education, 2014.

[40] Xie P., "A Web-based 3D Visualization Prototype System for High-resolution Satellite Colour Stereo Images," Tecnical Report, University of New Brunswick, 2004.

[41] Yang W., Zhang Z., Wang X., Moran B., Wheaton A., and Cooley N., Image Fusion and its Applications, InTech, 2011.

[42] Zebedin L., Klaus A., Gruber-Geymayer B., and Karner K., "Towards 3D Map Generation From Digital Aerial Images," ISPRS Journal of Photogrammetry and Remote Sensing, vol. 60, no. 6, pp. 413-427, 2006.

[43] Zhang B., "Automatic Spatial Modeler (ASM): Elevation by innovation," Tecnical Report, in Geospatial EXploitation Products, 2014.

[44] Zhang B., Miller S., DeVenecia K., and Walker S., "Automatic Terrain Extraction Using Multiple Image Pair and Back Matching," in Proceedings of ASPRS Annual Conference, Reno, 2006.

[45] Zhang B., Miller S., Walker S., and Devenecia K., "Next Generation Automatic Terrain Extraction Using Microsoft Ultracam Imagery," 
in Proceedings of ASPRS Annual Conference, Tampa, 2007.

[46] Zhang L., "Automatic Digital Surface Model (DSM) Generation from Linear Array Images," $\mathrm{PhD}$ Thesis, Institute of Geodesy and Photogrammetry Swiss Federal Institute of Technology (ETH) CH-8093 Zurich, 2005.

[47] Zhang L. and Gruen A., "Multi-Image Matching for DSM Generation from IKONOS Imagery," ISPRS Journal of Photogrammetry and Remote Sensing, vol. 60, no. 3, pp. 195-211, 2006.

[48] Zhao F., Huang Q., and Gao W., "Image Matching by Normalized Cross-Correlation," in Proceedings of IEEE International Conference on Acoustics Speech and Signal Processing Proceedings, Toulouse, pp. 729-732, 2006.

[49] Zitova B. and Flusser J., "Image Registration Methods: A Survey," Image and Vision Computing, vol. 21, no. 11, pp. 977-1000, 2003.

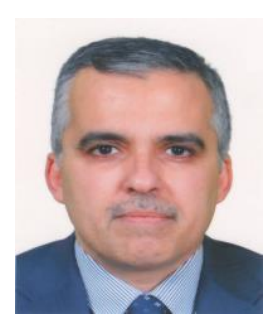

Haval Sadeq is a lecturer and head of the Geomatics (Surveying) Eng. dep. at Salahaddin University-Erbil, Iraq. $\mathrm{He}$ received his BSC degree fromSalahaddin University-Erbil, in 1996. He received his MSc degree in photogrammetry fromSalahaddin University-Erbil, Iraq, in 2000. In 2015 he received his $\mathrm{PhD}$ degree in Geomaticsfrom GlasgowUniversity, United Kingdom. His research interests are in photogrammetry, image matching, and, $3 \mathrm{D}$ city modelling from imagery. 